\title{
Numerical analysis of a mechanism of electrical conductivity of substance in the middle and lower mantle
}

\author{
L. N. Porokhova ${ }^{1}$, D. Yu. Abramova ${ }^{2}$, and D. A. Porokhov ${ }^{1}$ \\ ${ }^{1}$ Scientific-Research Institute of Physics, St. Petersburg State University, St. Petersburg 198904, Russia \\ ${ }^{2}$ IZMIRAN, Troitsk, Moskow 142092, Russia
}

(Received November 10, 1998; Revised July 13, 1999; Accepted July 14, 1999)

\begin{abstract}
The method proposed is based on the Arrenius' equation, that links electrical conductivity of solids with an enthalpy of activation, is studied and well-known distributions of temperature, pressure and electrical conductivity in the mantle. Formulas for the quantitative estimation of parameters, that characterize energy state of a lattice of the mantle, are received. Dependence of electrical conductivity on activation energy of free electron or ion, mobility of which has a power dependence on temperature, and of polaron with an exponential dependence on temperature was studied. The analysis of results has reveled that an electrical conductivity in the middle and in the lower mantle is realized by a change of activation energy of a polaron.
\end{abstract}

\section{Introduction}

Electrical conductivity of the mantle is one of the physical characteristics which are tightly bound both with properties of a lattice structure of a substance and with geothermal conditions to which the substance is posed.

There exist two main methods to study an abyssal electrical conductivity. One of them is to carry out laboratory experiments to determine electrical properties of a matter under high temperatures and pressures. Experimentally, it has been better studied an electrical conductivity of crust rock and the upper $400 \mathrm{~km}$ of the mantle. There is much less data about the middle $(670-1050 \mathrm{~km})$ and the lower of the mantle. The obstacle is that it is now technologically impossible to create a laboratory setup that could reproduce the conditions under which the mantle substance exists $(T>1500 \mathrm{~K}$, $P>20 \mathrm{GPa}$ with constant increase). Another method to gain the information is indirect one, but cheaper and more available - it is a use of electromagnetic probing methods. Published results of global and regional magnetovariational probing most of all give one information about a change of the electrical conductivity with depth in the upper and middle mantle. The distribution of the electrical conductivity in the lower mantle has been studied worse. There are only average estimations with large deviations of values, received from observations of secular variations of geomagnetic field produced by a source of internal origin. In the last years there have appeared papers, in which after an analysis of 7- and 11-year long variations of geomagnetic field from external sources, distributions of electrical conductivity in the lower mantle up to 2000-2500 km were given (Porokhova et al., 1996; Semenov, 1998). It has been confirmed, that an electrical conductivity in the mantle increases fairly fast with a depth. For a long time it was believed that the increase was

Copy right (C) The Society of Geomagnetism and Earth, Planetary and Space Sciences (SGEPSS); The Seismological Society of Japan; The Volcanological Society of Japan; The Geodetic Society of Japan; The Japanese Society for Planetary Sciences. monotonic, and it was associated with an increase of a heat energy. Along with a progress on probing techniques, methods of data processing and interpretations of results, there appeared qualitatively new data that allowed constructions of new models of an electrical conductivity of the mantle. Some researchers (Oraevskiy et al., 1993; Semenov et al., 1997; Semenov, 1998) believe that in the middle mantle there exists a conductive layer with pretty distinctive borders. Others (Constable, 1993; Shultz et al., 1993; Porokhova et al., 1996) think that in the middle mantle an electrical conductivity smoothly approaches a constant value thus forming a plateau. Most probable, further elaborations of models will remove remaining uncertainties. Essential disagreements are related to only $400-500 \mathrm{~km}$ of the whole depth of the mantle and can not be an obstacle for a use of experimental data in studying of conductive properties of a mantle substance.

\section{Electronic or Ionic Electrical Conductivity}

According to modern views, the substance of the mantle consists of solid crystal compounds that belong to a family of semiconductors and dielectrics with permanent chemical structure in the middle and lower mantle (Brown and Mussett, 1984).

For any way of charge transfer, the link between an electrical conductivity of solids and an activation enthalpy is described by the equation:

$$
\sigma=\sigma_{0} \cdot \exp \left[-\frac{\Delta H}{k_{B} T}\right],
$$

where $T$ is a temperature at which the substance is, $k_{B}$ is the Boltzmann's constant;

$$
\Delta H=\Delta U+P \Delta V,
$$

$\Delta U$ is an activation energy (a height of potential barrier), $P$ is a pressure, $\Delta V$ is an activation volume, that reflects 
an influence of a pressure on an energy barrier to charge transport.

Pre-exponential coefficient in (1) is

$$
\sigma_{0}=n \cdot q \cdot \mu
$$

where $n, q, \mu$ are the amount, the charge and the mobility of charge carriers respectively.

Let us first assume that an electrical conductivity is produced by jump-like movements of free electrons or ions. For both of the mechanisms the mobility has the power dependence on a temperature:

$$
\mu=\frac{a^{2} v}{6 k_{B} T}
$$

where $a$ is a distance between two equilibrium positions (the length of a jump), $v$ is a jumping rate.

The aim is to find quantitative estimations of $\sigma_{0}, \Delta H$, $\Delta U, \Delta V$ for different depths in the middle and lower mantle, with the values of temperature $T$, pressure $P$ and electrical conductivity $\sigma$ given. Pre-exponential coefficient $\sigma_{0}$ and an activation energy $\Delta U$ characterize conductive properties of a matter while $T$ and $P$ characterize the thermodynamic conditions.

For solving the problem we took the logarithm of both sides of the Eq. (1):

$$
\ln \sigma=\ln \sigma_{0}-\frac{\Delta H}{k_{B} T} .
$$

Substituting $\sigma_{0}$ by formulas (3) and (4), we differentiate the obtained expression over $\left(k_{B} T\right)^{-1}$ and over $P$ :

$$
\frac{\partial \ln \sigma}{\partial\left(k_{B} T\right)^{-1}}=k_{B} T-\Delta H, \quad \frac{\partial \ln \sigma}{\partial P}=-\frac{\Delta V}{\left(k_{B} T\right)} .
$$

Taking into account known transformations

$$
\frac{\partial \ln P}{\partial P}=\frac{1}{P}, \quad \frac{\partial \ln \left(k_{B} T\right)}{\partial \frac{1}{\left(k_{B} T\right)}}=-k_{B} T
$$

and using (2) and (5) we receive the final formulas for further analysis of the mechanism of electrical conductivity of the substance in the mantle:

$$
\begin{gathered}
\Delta U=k_{B} T \cdot\left[\frac{\partial \ln \sigma}{\partial \ln P}+\frac{\partial \ln \sigma}{\partial \ln \left(k_{B} T\right)}+1\right] \\
\Delta V=-\frac{k_{B} T}{P} \cdot\left[\frac{\partial \ln \sigma}{\partial \ln P}\right] \\
\Delta H=k_{B} T \cdot\left[\frac{\partial \ln \sigma}{\partial \ln \left(k_{B} T\right)}+1\right] \\
\ln \sigma_{0}=\ln \sigma+\left[\frac{\partial \ln \sigma}{\partial \ln \left(k_{B} T\right)}+1\right] .
\end{gathered}
$$

The formulas (7) are written down for a fixed depth. We have carried out calculations for depth ranging from 500 to $2900 \mathrm{~km}$ with the step of $100 \mathrm{~km}$ and have studied changes of values considered with depth as temperature and pressure grew. The distributions of temperatures we took from (Shankland and Brown, 1985), one of pressure we took from the PREM model (Dziewonski and Anderson, 1981), and one of electrical conductivity took from a model, constructed on a basis of a joint interpretation of satellite (MAGSAT) and ground data on a global magnetovariational probing (Porokhova et al., 1996).

The results are presented on Figs. 1 and 2. The enthalpy of activation $\Delta H$ is less than $\Delta U$ at all the explored depths (see Fig. 1). According to the formula (2), the reason is negative values of an activation volume, that agrees with the electronic mechanism of the electrical conductivity. If the electrical conductivity was ionic, one should expect positive values of $\Delta V$ since the ionic movement is accompanied with an expansion of a crystal lattice, like in a case of diffusion.

At about $2500 \mathrm{~km}$ an activation energy has abnormal large jump that most likely points to a break of the validity of the Arrenius' equation in the domain of the transfer from the mantle to the core. So in what follows we will discuss results related to depths up to $2300 \mathrm{~km}$.

According to (1)-(4), the behavior of the electrical conductivity of free electrons is determined most of all by the exponential term. However, the analysis of the results shows (see Fig. 2) that at depths 500-700 and 1200-2400 km the exponent is constant and the electrical conductivity grows proportionally to $\sigma_{0}$. Only at depths of about $700-1200 \mathrm{~km}$ due to the influence of the exponent, the increase of the electrical conductivity slows down and a value of the electrical conductivity gets stable. Obviously, the hypothesis of a free electrons charge transport is not confirmed by the experimental data. Most likely, the fast increase of $\sigma_{0}$ is linked with an

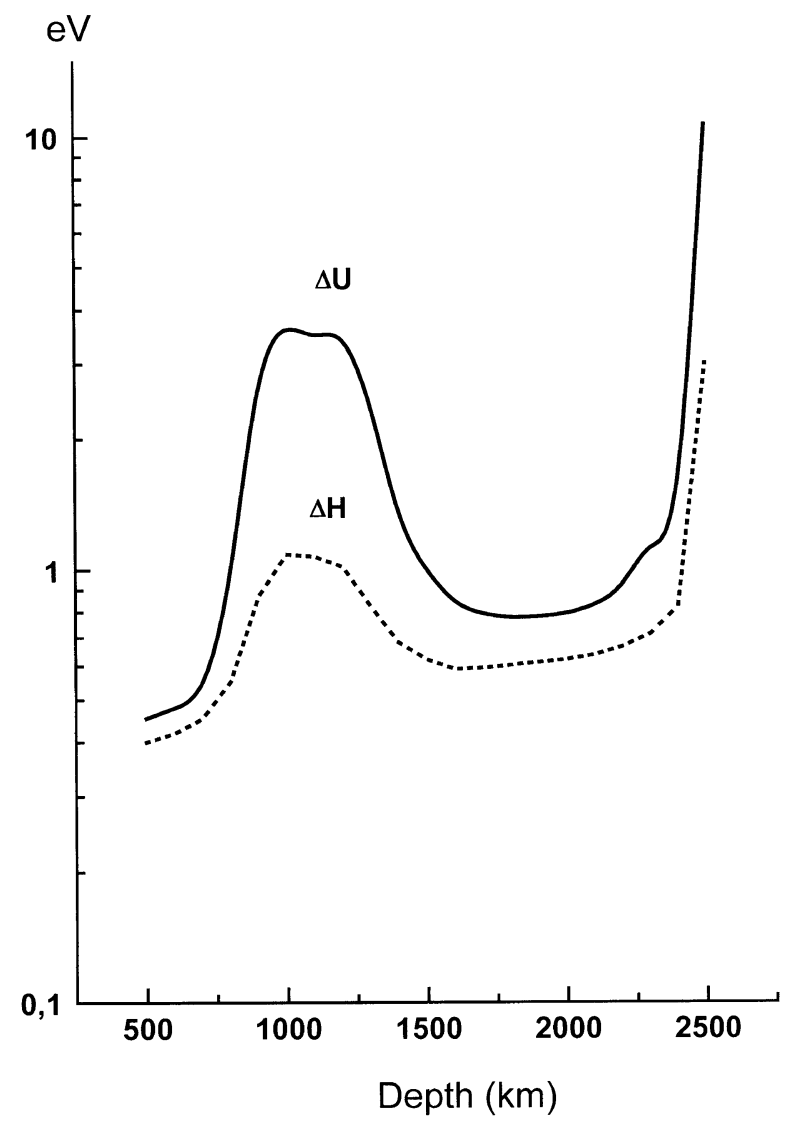

Fig. 1. Varying of the energy and enthalpy of the activation for the depths of middle and lower mantle. 


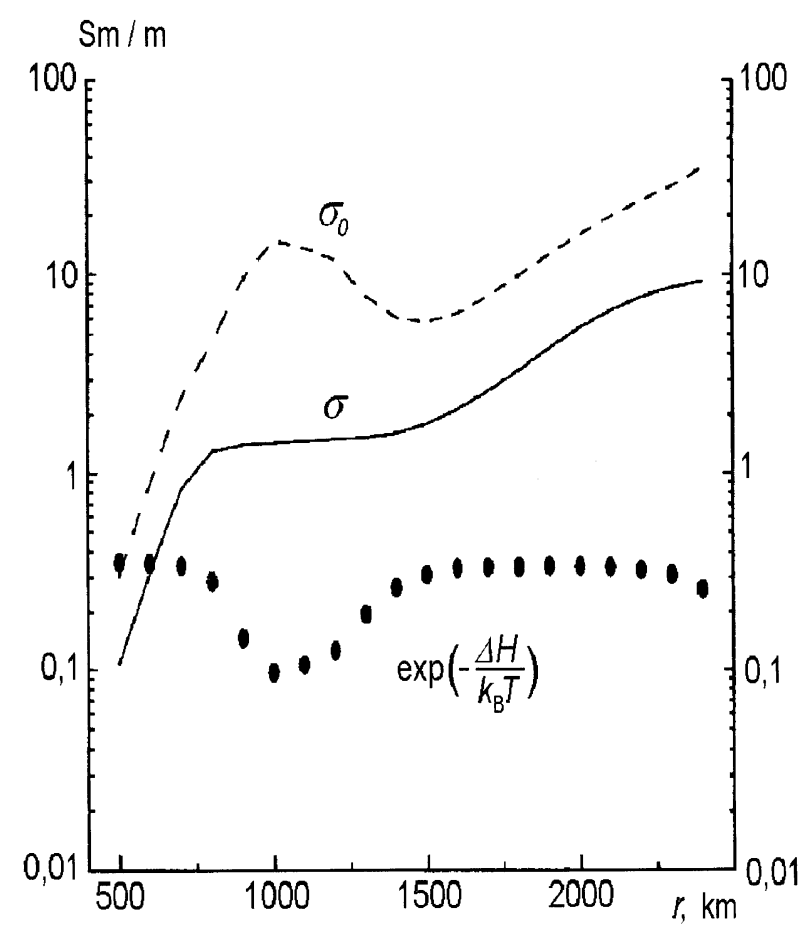

Fig. 2. The global model of electrical conductivity and values of co-factor of Arrenius' equation obtained using this model with power-kind temperature dependence of the mobility of the charge carrier.

influence of lythostatic pressure, that grows with a depth, on a crystal lattice under huge temperatures. The idea of priority influence of the pressure has been already suggested as well (Parhomenko and Bondarenko, 1972; Zharkov, 1983).

\section{Polaronic Electrical Conductivity}

There is no explicit dependence between macro- and micro-processes that take place in the mantle. One can only suppose that under conditions of high temperatures and pressures, the crystallic lattice of the mantle substance gets distorted, and that leads to an increase of amount and mobility of charge carriers.

The latter is typical for a polaron jumping mechanism of conductance under high temperatures (polaron of a small radius). Let us recall that the polaron conductance appears only in the case when electrons are tightly bound with heat oscillations of ionic crystallic lattice (phonons). In ionic crystals, electrons polarize a certain area around them and get localized in it. This area of distorted lattice (polaronic) together with the electron in it, that causes the distortion, received a term polaron. Due to external influence under conditions of high temperatures, a polaron moves across a cry stall by means of jumping mechanism. Its mobility grows exponentially with temperature:

$$
\mu_{p} \approx a_{p}{ }^{2} \cdot v_{p} \cdot \exp \left[-\frac{\Delta E_{p}}{k_{B} T}\right],
$$

where $\Delta E_{p}$ is the energy necessary for a creation of the polaronic (empty) area; $a_{p}$ is a constant of lattice, or the average distance between atoms; $v_{p}$ is a frequency of phonons.

The activation energy of a polaron $\Delta U_{1}$ consists of two parts:

$$
\Delta U_{1}=\Delta E_{p}+\Delta U_{p}
$$

where $\Delta U_{p}$ is the energy of electron which is localized in polaronic area created by the electron. The activation enthalpy of a polaron

$$
\Delta H_{p}=\Delta U_{1}+P \Delta V_{p}
$$

The process of polaronic charge transfer is described by the equation

$$
\sigma=A \cdot \exp \left[-\frac{\Delta H_{p}}{k_{B} T}\right] .
$$

This equation like as the Eq. (1). But such a similarly is related only to the forms of the equations. The physical meanings of the variables are different. So, the pre-exponential coefficient in (9) $A=n_{p} a^{2} q v_{p}$ is a constant value $\left(n_{p}\right.$ is the number of polarons). It is independent on the temperature unlike to the pre-exponential coefficient $\sigma_{0}$ in (1), which is dependent on temperature as it is seen from (3) and (4). The activation energy of a polaron $\Delta U_{1}$ means the activization energy of a "jump" but not the height of the potential barrier as it is in the case a free electron. The activation volume $\Delta V_{p}$ in (9) means the variation of the crystallic lattice volume taking place in the process of a polaron creation.

Generally speaking, the Eq. (9) has only one unknown variable $\Delta U_{1}$ since $\Delta V_{p}=-k_{B} T(\partial \ln \sigma / \partial P)$. The value of $A$ is equal to $\sigma$ in the limit of $\left(k_{B} T\right)^{-1}$ running to zero. For experimental electrical conductivity $\ln A$ is seen to be less than or equal to 3 (see Fig. 3). Then the activation energy of

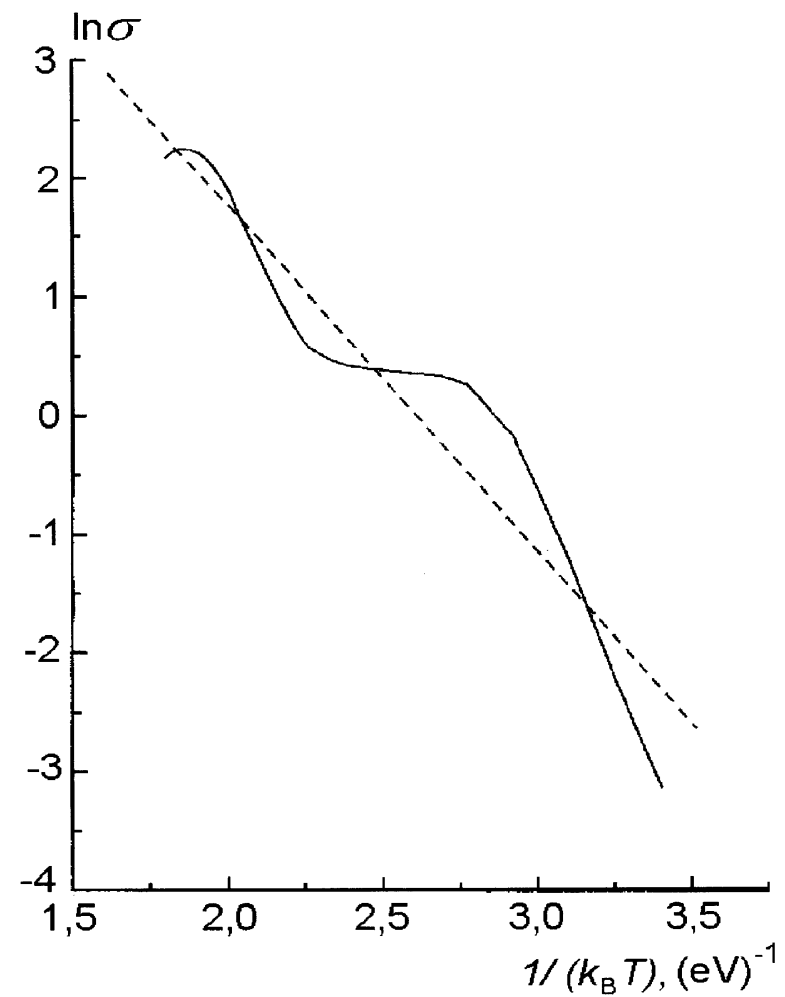

Fig. 3. The dependence of the conductivity on the temperature. The primed line is the result of approximation of the function $\sigma\left(\frac{1}{k_{B} T}\right)$. 


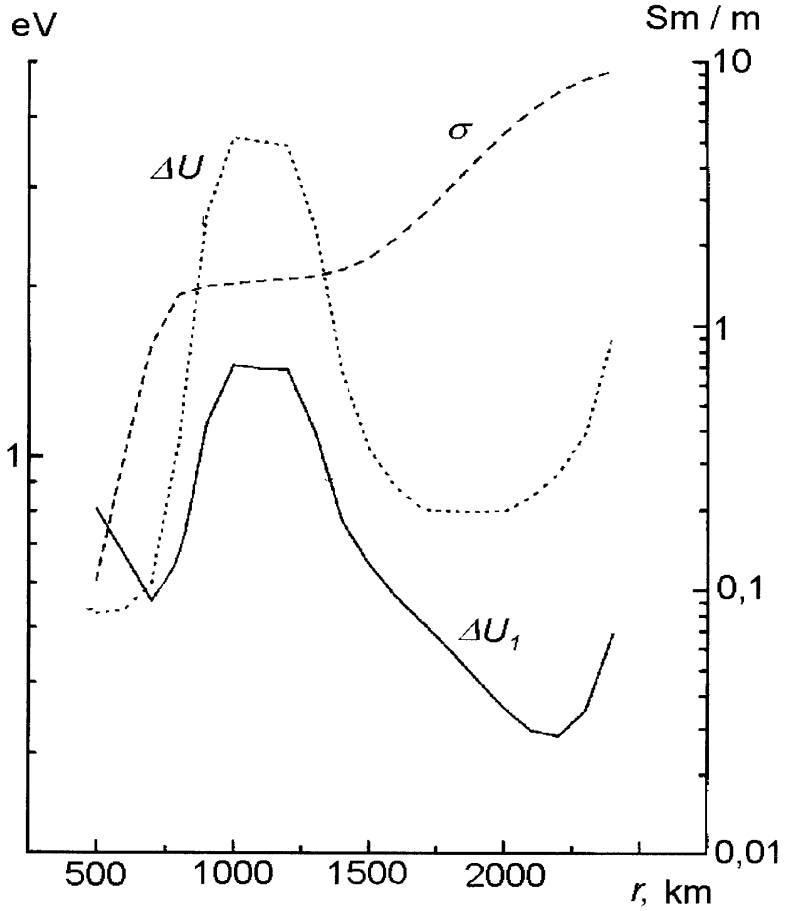

Fig. 4. Varying of the activation energy at the mantle depths over two mechanism of charge carry: polaron (localized electron $\Delta U_{1}$ ), and free electron $(\Delta U)$

a polaron is

$$
\Delta U_{1}=k_{B} T\left[\ln A-\ln \sigma-\frac{P \Delta V_{p}}{k_{B} T}\right] .
$$

The plot of the dependence of $\Delta U_{1}$ on depth is presented on Fig. 4. For comparing there is also presented the behavior of an activation energy of a free electron $\Delta U$. First of all, it pay attention that jumps of a localized electron demand less energy that ones of a free electron. It would be linked with that due to the high temperatures in the mantle the magnitude of heat oscillations is very large and a free electron needs more energy to overcome the oscillations. Activation energy of both of the charge carriers changes with depth jump-like, with jumps observed at the same depths. Comparison of the $\Delta U$ and $\Delta U_{1}$ with the plot $\sigma(r)$, presented on the same figure, shows that the electrical conductivity in the mantle is realized by a change of an activation energy of a polaron.

How far one can trust the conclusions made? It seems impossible yet to give a quantitative estimation for each value received, for there is no quantitative estimations of the data on temperatures and pressures used. We carried out calculations with several models of distributions of thermodynamic parameters, which were presented in different publications (Stacey, 1972; Zharkov, 1983; Brown and Mussett, 1984), for the same model of electrical conductivity. No principal differences were found. Chosen model of global distribution of electrical conductivity in the middle and lower mantle meets fairly well (see Fig. 5) the results of laboratory studies performed by (Shankland et al., 1993). That is why we preferred the very model. Assurance in rightfulness of our results would strengthen if they don't contradict the modern views about a mantle structure. Let us turn to Fig. 4. The

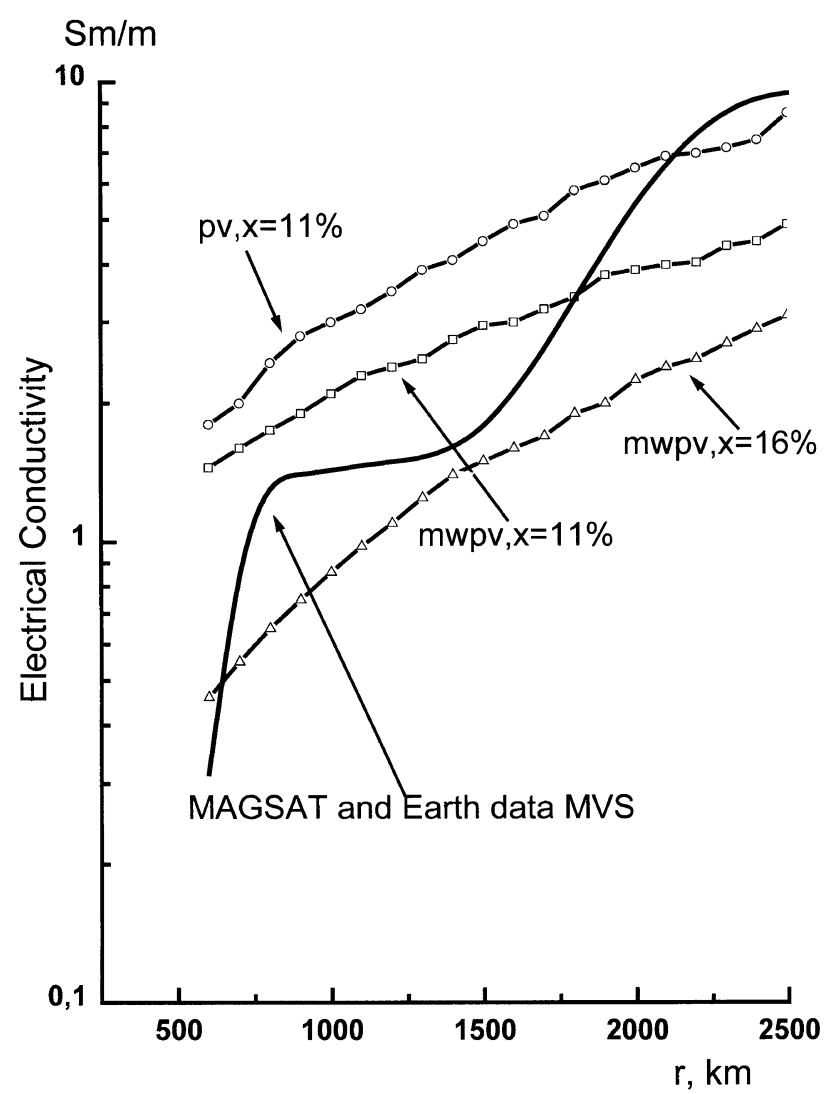

Fig. 5. Comparison of the electrical conductivity of middle and lower mantle obtained after interpretation of joint ground and satellite data (Porokhova et al., 1996) with laboratory experiments (Shankland et al., 1993) where $p v, x=11 \%$ is perovskite with an iron content of 11 atom $\% ; m w-p v$, $x=11 \%$ and $x=16 \%$ is magnesiowustite perovsite with an iron content of 11 and 16 atom $\%$ accordingly.

curve of the activation energy behaves jump-like. The jumps, most likely linked with phase transitions, are observed at the depths of 700 and $1200 \mathrm{~km}$. Let us note that at $700 \mathrm{~km}$, according with gravimetric data, there occurs a change of a sign of excess density, and at about 670 and 1050 there are borders fixed by seismologists. The activation energy between the jumps is constant. It would mean that during phase transitions, occurring at the depths, a change of a structure and a density of a substance takes place, but without any change in chemical structure. Indeed, a significant raise of density at $650 \mathrm{~km}$ and a small increase at $1050 \mathrm{~km}$ were noticed in (Brown and Mussett, 1984). The curve of the activation energy has one more jump at depths about 2400-2500 km (see Fig. 1). It is known that at about the same depths, a velocity of transversal waves drops, features of non-linear grow of a density appears (Brown and Mussett, 1984). Abnormal large values of activation energies allow one to suppose an existence in the base ground of the mantle an intermediate zone, witch consists of a substance with a chemical structure different from the one of the upper-laying layers. Such coherence of the results, received by independent geophysical methods, verifies rightfulness of our approach to studying of conductive properties of a mantle substance, thus allowing one to narrow a range of varying the substance's properties. 


\section{Conclusion}

In the last years, the question of possible polaron mechanism of the electrical conductivity at depths of 670-2880 $\mathrm{km}$ has been often discussed in literature, basing on results of laboratory experiments (Constable et al., 1992; Hirch et al., 1993; Shankland et al., 1993). Here, for the first time, we made an attempt to use measurements of global magnetovariational probing for the study of a mechanism of an electrical conductivity at unavailable depths. However, it should be kept in mind that the Eqs. (1) and (7) are valid for a matter with a constant chemical structure. In laboratory studies, this condition is thoroughly fulfilled. As to the mantle, there is no absolute guarantee (Brown and Mussett, 1984). Most widely accepted now is the hypothesis of the uniform chemical structure of the middle and lower mantle. Nevertheless, one can not eliminate the possibility of chemical non-uniformity of the mantle. In that case there could occur another micro processes responsible for the electric properties of the mantle.

Acknowledgments. The authors wish to thank professor Tom Shankland (Los Alamos Laboratory, USA) for his interest to our research and discussion of problem questions, and also professor $\mathrm{E}$. V. Charnaya (St. Petersburg State University, Russia) for her fruitful advises in analysis of the results and for preparing of the paper.

This work was supported by the Ministry of High and Professional Education, grant 97-13-27, the Competitive Centre of Fundamental Research.

\section{References}

Brown, G. C. and A. E. Mussett, The Inaccessible Earth, 260 pp., Mir, Moscow, 1984 (in Russian).

Constable, S., T. J. Shancland, and A. G. Duba, The electrical conductivity of an izotropic olivine mantle, Geophys. Res., 97(B3), 3397-3404, 1992. Constable, S., Constraints on mantle electrical conductivity from field and laboratory measurements, J. Geomag. Geoelectr., 45, 707-728, 1993.

Dsiwonski, A. M. and D. L. Anderson, Preliminari reference Earth model, Phys. Earth Planet. Inter., 25, 297-356, 1981.

Hirch, L. M., T. J. Shancland, and A. G. Duba, Electrical conduction and polaron mobility in Fe-bearing olivine, Geophys. J. Int., 114, 36-44, 1993.

Oraevsky, V. N., N. M. Rotanova, V. I. Dmitriev, E. N. Bondar, and D. Yu. Abramova, Results of deep magnetovariational sounding of Earth using ground data and satellite measurements ("MAGSAT"), Geomagn. aeron., 33(2), 120-127, 1993 (in Russian).

Parhomenko, A. I. and A. T. Bondarenko, The Electrical Conductivity of Rocks under High Pressures and Temperatures, 278 pp., Nauka, Moscow, 1972.

Porokhova, L. N., D. Yu. Abramova, and D. A. Porokhov, Models of the mantle electrical conductivity constructed be the method of Efficient Linearization using the global ground and satellite data, Geomagn. aeron., 36(5), 228-237 1996 (in Russian).

Semenov, V. Yu., Regional Conductivity Structures of the Earth's Mantle, 119 pp., Publis. Inst/geophys. Pol. Acad. Sc., C-65(3-2), 1998.

Semenov, V. Yu. and E. P. Harin, The electrical conductivity of the mantle using data of Russian observatory, Physics of Earth, 9, 31-37, 1997 (in Russian).

Shankland, T. J. and Brown, J. M., Homogeneity and temperatures in the lower mantle, Phys. Earth Planet. Inter, 38, 51-58, 1985.

Shankland, T. J., J. Peyronneu, and J. P. Polrler, Electrical conductivity of the Earth's lower mantle, Montly Nature, 1(12), 80-82, 1993.

Shultz, A. and V. Yu. Semenov, Modelling of the structure of electrical conductivity of the middle Earth' mantle, Physics of Earth, 10, 39-43, 1993 (in Russian).

Stacey, F. D., Physics of the Earth, 342 pp., Mir, Moscow, 1972 (in Russian). Zharkov, V. N., Internal Structure of Earth and Planets, 191 pp., Nauka, Moscow, 1983.

L. N. Porokhova (e-mail: Ludmila.Porokhova@pobox.spbu.ru), D. Yu. Abramova, and D. A. Porokhov 